\title{
Calorie Restriction Mimetics: Examples and Mode of Action
}

\author{
Marios Kyriazis*
}

British Longevity Society, UK

\begin{abstract}
The search for Calorie Restriction Mimetics (CRM) - compounds that mimic the genetic, biochemical and physical actions of calorie restriction - is not a search for a 'lazy dieters pill'. It is a quest aiming to clarify the basic mechanisms of calorie restriction and develop strategies in order to prevent, treat or alleviate age-related conditions. The development of CRM will add new and important assets in our armamentarium of anti-ageing therapies, with the ultimate result of increasing healthy human lifespan. This Special Supplement on CRM is an attempt to discuss some agents which may be used instead of calorie restriction itself. Agents such as resveratrol, metformin, carnosine and Rimonabant are mainstream oral therapies already used by millions of people for other clinical indications. New CRM such as NADH, gugulipids and certain drugs that interfere with glucose metabolism can be also used as oral therapy. Less easily available CRM such as oxaloacetic acid, naloxone, leptin, adiponectin, rapamycin and sirtuins are further examples of promising agents. In order for the therapy to be effective, a combination of these must be used. This paper summarises the actions of calorie restriction and then suggests several examples of possible CRM. Some of these examples can be used in everyday clinical setting.
\end{abstract}

Keywords: Calorie restriction, calorie restriction mimetics, health-span, hormesis.

\section{INTRODUCTION}

Calorie restriction (CR) is discussed elsewhere in this Supplement. Its practical aim is not only to increase average and maximum lifespan in humans, but also to prolong the 'health-span' which is the number of years an organism can live without any major chronic diseases [1]. It may be somewhat simplistic but practically useful to divide the effects of CR into three general categories: genetic, biochemical and physical. The following list is by no means exhaustive but highlights some examples.

A. Genetic. These are effects at specific gene level which can modulate transcription of enzymes or other factors. Perhaps the most promising CRM are those which work on this level.

- Decreases the activity of p53 [2], and therefore modulates apoptosis [3].

- Regulates Sir-2 [4] and activates Sirt1 [5]. Sirt1 is activated to promote transcription of genes that deal with the stress response and adaptation.

- $\quad$ Regulates Daf-16. AMPK (AMP Protein Activated Kinase) is activated in the presence of Daf-16 [6].

B. Biochemical effects are those that directly influence macromolecules, without an identifiable genetic origin:

- Reduces lipid peroxidation and generation of superoxides [7].

- $\quad$ Reduces iNOS expression and COX2 expression [8], and increases NADH concentration within the mitochondria [9].

*Address correspondence to this author at the British Longevity Society, UK; E-mail: drmarios@live.it
- $\quad$ Maintains DHEA levels [10].

- $\quad$ Modulates PPAR [11]. Suppresses PGE-2, TNF-alpha and CRP (thus reduces the inflammation response) [12].

- $\quad$ Stimulates Brain-Derived Neurotropic Factor (BDNF) [13].

C. Physical changes include clinically relevant and measurable parameters, at the organismic level.:

- $\quad$ Reduces body weight and body temperature [14].

- Improves diastolic function. Lowers cholesterol, blood pressure and pulse rate, and reduces blood glucose levels [15].

- Increases muscle mass and reduces fat mass (including intra-abdominal fat) [16].

- Improves memory and cognition $[17,18]$.

\section{CANDIDATE CRM}

Calorie restriction mimetics (CRM) are drugs or chemical compounds which mimic the actions of CR. It is not sufficient for a compound that mimics just one effect of CR to be classified as a CRM. As an arbitrary guide, I propose that, in order for a compound to be classified as a CRM, it has to mimic at least two biochemical plus one genetic, or five biochemical/physical effects of CR. This is a general attempt at defining a CRM and further discussion is needed, although initial attempts along these lines have already been made [19], and in particular with regards to defining biomarkers in calorie restriction has already taken place [20]. Examples of CRM that are already available and currently used for other indications are: 


\section{Metformin}

Metformin is a receptor sensitizer, because it enhances the sensitivity of insulin receptors on the surface of muscle and fat cells [21]. It can activate genes which reduce hepatic production of glucose, thus reducing the risk of glycation and other age-related damage. In addition, metformin reduces gene expression of enzymes which increase oxidation of fatty acids. Further research is needed to clarify the approximate dose of metformin for CRM effects. Healthy people who take metformin for its general anti-ageing benefits use $500 \mathrm{mg}$ twice a day. Side effects may include gastrointestinal problems and allergic reactions.

\section{Resveratrol}

This is a polyphenol with proven beneficial cardiovascular effects and a potent CRM [22]. In yeast, it stimulates Sir2 (silent information regulator), increasing DNA stability and extending life-span by $70 \%$. Resveratrol activates the human homologue SIRT1 which results in reduced apoptosis in the liver, blood and skin, and reduced risk of age-related chronic disease. The dose of resveratrol is normally between $5 \mathrm{mg}$ and $15 \mathrm{mg}$ daily, however the dose necessary to achieve CRM effects has not yet been calculated. Long term adverse effects are unknown, but no significant short term side effects have been reported.

\section{Rimonabant (Acomplia)}

Endocannabinoids are cannabis-like chemicals which stimulate appetite and regulate energy balance. Overstimulation of endocannabinoid receptor in the hypothalamus promotes appetite and stimulates lipogenesis [23]. It also blocks adiponectin. Rimonabant (an anti-obesity drug) is an endocannabinoid-1 receptor blocker, which reduces appetite, balances energy production and increases adiponectin which, in turn, reduces intra-abdominal fat [24]. Rimonabant improves lipid profile, glucose tolerance, and waist measurement. Therefore, it has effects similar to those of CR. It is taken 20 mg once daily, preferably with a mild calorie restricted diet. The efficacy and long term adverse effects of rimonabant have recently been questioned and further research is needed to clarify these.

\section{Anti-Glycators}

Agents which reduce abnormal protein accumulation (aminoguanidine and carnosine) can also be CRM. These prevent glycation and therefore reduce AGEs (Advance Glycation End-products) formation [25]. AGEs contribute to extensive age-related damage such as accumulation of amyloid-beta implicated in Alzheimer's disease. CR reduces the concentration of AGEs. The same mechanism is shared by aminoguanidine and carnosine which prevent and eliminate AGEs, therefore contributing towards the prevention of chronic degenerative disease. No significant side effects have been reported, and mild gastrointestinal problems usually improve after reducing the dose.

\section{Exendin}

The agent exendin (exanatide, exanadin) reduces plasma glucose, suppresses food intake and regulates glucose metabolism [26]. It is a GLP (Glucagon-Like Peptide) modulator, able to increase brain function and protect the brain against toxicity. Exanatide $\left(\right.$ Byetta $\left.^{\circledR}\right)$ was approved by the Food and Drug Administration for treatment of Type 2 Diabetes in patients who are already on an oral diabetes medication. The product comes in pre-filled syringes and is injected subcutaneously twice daily.

\section{Olbetam (Acipimox)}

This agent inhibits the release of fatty acids from adipose tissue and reduces blood concentration of very low density lipoproteins and low density lipoproteins with a subsequent reduction in triglyceride and cholesterol levels [27]. It improves growth hormone secretion and reduces lipid peroxidation. Olbetam is indicated as adjunctive therapy to diet and weight loss in the treatment of several lipid disorders. The dosage is between $500-750 \mathrm{mg} /$ day.

\section{PPAR Gamma Modulators}

Peroxisome proliferator-activated receptors (PPARs) are members of the nuclear hormone receptor superfamily of transcription factors that are related to retinoid, steroid and thyroid hormone receptors. PPARs play an important role in many cellular functions including lipid metabolism, cell proliferation, differentiation, adipogenesis and inflammatory signaling. Modulation of PPAR gamma generally reduces inflammation, improves immunity and reduces blood glucose. Two examples of PPAR modulators are:

a) Rosiglitazone (Avantia), an insulin-sensitizing drug that is a ligand for PPAR-gamma [28]. The dose is 4 $\mathrm{mg}$ once or twice a day.

b) Gugulipids, from the plant Commiphora mukul, which block the PPAR-mediated differentiation of preadipocytes into mature adipocytes [29].

Two very promising CRM (NADH/oxaloacetic acid and Naloxone) are discussed elsewhere in this Supplement. Other candidate CRM which are not readily available are listed below. The list is merely an example of possible CRM and it is by no means exhaustive. Further research regarding the CRM effects of each compound may help in establishing their mode of action. Furthermore, the clinical adverse effects of these agents have not been clarified and are best used under expert specialist supervision.

\section{Leptin}

This is a molecule, produced by adipocytes, that stimulates fat metabolism and reduces body weight. A reduction of dietary intake causes leptin levels to fall and this interferes with the secretion of testosterone, progesterone, growth hormone and thyroid hormones as a response for adaptation [30]. Therefore, leptin mediates the clinical effects of CR. As a result, agents which affect leptin production must also be classified as CRM. Together with insulin and ghrelin (a growth hormone stimulator) leptin balances the ratio of appetite promoters vs. appetite blockers in the hypothalamus in the brain and so regulates homeostasis and food intake. Leptin is stimulated by PPAR modulators such as rosiglitazone. Human recombinant leptin costs approximately $\$ 140$ for $0.5 \mathrm{mg}$, but nicotinic acid can help increase its concentration. However, leptin mediates the effects of diabetic cardiomyopathy [31] and its long term effects are not clear. 


\section{Deoxyglucose}

The first CRM described, inhibits glycolysis and mimics some of the effects of CR, particularly increased insulin sensitivity, reduced glucose levels and other biochemical changes [32]. Research is still under way to identify more about its possible benefits on humans. What is known about deoxyglucose is that it can be toxic in high dosages.

\section{Modulators of Sirtuins}

Sirtuins are histone deacetylases that catalyze deacetylation reaction in an $\mathrm{NAD}(+)$-dependent manner [33]. Activation of sirtuins improves longevity and health span in many species. This can be achieved by STAC -sirtuin activating compounds. Examples of STAC are chalchone [34], sirtinol, which among other actions, reduces pro-inflammation mediators [35] and fisetin (a flavonoid, antioxidant compound). Fisetin is a potent suppressor of some inflammatory cytokines/chemokines and an angiogenic factor [36].

\section{4-Phenylbutyrate (PBA)}

Increases median and maximum lifespan in flies. In addition, it increases histone deacetylation [37].

\section{Hydroxycitrate}

An active ingredient extracted from the Garcinia cambogia, reduces caloric intake and cholesterol [38] and it is currently used in weight control [39].

\section{Gymnemoside}

Isolated from the leaves of Gymnema sylvestre [40], gymnemoside modulates glucose metabolism.

\section{Adiponectin}

Together with leptin, it takes part in fat metabolism. It is activated by PPAR modulators such as rosiglitazone [41]. It enhances phosphorylation of AMPK [42], although it can increase total and cardiovascular mortality [43]. Human recombinant adiponectin is available for sale costing approx $\$ 350$ per $50 \mathrm{mcg}$.

\section{Iodoacetate}

An alkylating agent, it protects against toxic metabolites of glucose. Iodoacetate prevents formation of disulfide bonds, is a glycolysis-inhibitor and an anti-cancer agent [44].

\section{DPP-4 Inhibitors}

Diapeptidyl peptidase-4 (DPP-4) is an enzyme that modulates Glucagon-Like Peptide, allowing glucagon to increase glucose concentration [45]. DPP-4 inhibitors have the opposite effect, reducing glucose plasma levels, and are candidate CRM.

\section{Peptide PYY3-36}

This protein fragment is released from the bowel following a meal. It then inhibits food intake by acting on the hunger centre in the hypothalamus [46]. By reducing appetite and glucose metabolism (actions similar to those seen in $\mathrm{CR}$ ), it can meet some of the criteria for consideration as a CRM.

\section{Modulators of NPY}

The neuropeptide Y (NPY) is a small protein fragment which increases appetite, induces obesity and reduces the metabolic rate. CR modulates the production of NPY by selectively blocking receptors in the hippocampal region of the brain and by stimulating others in the hypothalamus. Any modulation of NPY release would result in exactly the same clinical effects as those seen in CR [47].

\section{Rapamycin}

It is known that CR downregulates TOR (target of rapamycin) [48]. Many longevity genes encode components of TOR pathway, so rapamycin which is a TOR inhibitor, can be classified as a CRM.

\section{Galanin Antagonists}

These block galanin (which increases appetite and reduces insulin) [49,50]. An example is the antagonist M35.

\section{Aldifen (2,4-Dinitrophenol)}

is a hormetic metabolic poison [51] that causes mild mitochondrial uncoupling, interfering with energy production. However, overdose is lethal. Nevertheless, metabolic poisons with hormetic effects (such as oligomycin, carbonylcyanide, rotenone, antimycin, and malonate) are being investigated as having not only CRM actions but also other health benefits [52].

\section{GENERAL CONCLUSIONS}

Clearly, a CRM cannot mimic all of the actions of CR, so it must be combined with other CRM which complement each other, in order to cover as many actions of CR as possible.

For example, Acomplia, metformin and resveratrol can be combined for maximum effect. It is worth noting that Intermittent Fasting (IF) is an intervention that may mimic the effects of CR itself. However, IF increases lifespan even when the overall calories are not reduced. It appears that it is the stress of fasting rather than the reduced calories that cause the benefit. This supports the view that the stress of fasting is a hormetic challenge which helps activate the protection pathways that are also active in CR [53]. A search for CRM can be extended to find IF mimetics such as RHEB, a GTPase, which is a mediator through Daf16 and TOR [54].

The increased amount of research into $\mathrm{CR}$ has given us promising directions into identifying effective agents which reproduce the exact benefits of $\mathrm{CR}$, without the need to follow long calorie-restricted diets. The most promising and clinically relevant CRM are those that reproduce at least one genetic and two biochemical, or at least five biochemi$\mathrm{cal} /$ clinical benefits of CR. While research is continuing, many physicians who already recommend these compounds to their patients for other indications, have now started realising that their treatment has an added possible healthextending bonus.

\section{REFERENCES}

[1] Barzilai N, Bartke A. Biological approaches to mechanistically understand the healthy life span extension achieved by calorie restriction and modulation of hormones. J Gerontol A Biol Sci Med Sci 2009; 64(2): 187-91. 
[2] Bauer JH, Poon PC, Glatt-Deeley H, Abrams JM, Helfand SL. Neuronal expression of p53 dominant-negative proteins in adult Drosophila melanogaster extends life span. Curr Biol 2005; 15(22): 2063-8.

[3] Marzetti E, Lawler JM, Hiona A, Manini T, Seo AY, Leeuwenburgh C. Modulation of age-induced apoptotic signaling and cellular remodeling by exercise and calorie restriction in skeletal muscle. Free Radic Biol Med 2008; 44(2): 160-8.

[4] Allard JS, Perez E, Zou S, de Cabo R. Dietary activators of Sirt1. Mol Cell Endocrinol 2009; 299(1): 58-63.

[5] Jiang WJ. Sirtuins: novel targets for metabolic disease in drug development. Biochem Biophys Res Commun 2008; 373(3): 341-4.

[6] Greer EL, Dowlatshahi D, Banko MR, et al. An AMPK-FOXO pathway mediates longevity induced by a novel method of dietary restriction in C. elegans. Curr Biol 2007; 17: 1646-56.

[7] Hyun DH, Emerson SS, Jo DG, Mattson MP, de Cabo R. Calorie restriction up-regulates the plasma membrane redox system in brain cells and suppresses oxidative stress during aging. Proc Natl Acad Sci USA 2006; 103(52): 19908-12.

[8] Kim YJ, Kim HJ, No JK, Chung HY, Fernandes G. Antiinflammatory action of dietary fish oil and calorie restriction. Life Sci 2006; 78(21): 2523-32.

[9] Yang H, Yang T, Baur JA, et al. Nutrient-sensitive mitochondrial NAD+ levels dictate cell survi. J Clin Endocrinol Metab 1997; 82(7): 2093-6.

[10] Lane MA, Ingram DK, Ball SS, Roth GS. Dehydroepiandrosterone sulfate: a biomarker of primate aging slowed by calorie restriction. J Clin Endocrinol Metab 1997; 82(7): 2093-6.

[11] Mulligan JD, Stewart AM, Saupe KW. Downregulation of plasma insulin levels and hepatic PPARgamma expression during the first week of caloric restriction in mice. Exp Gerontol 2008; 43(3): 14653.

[12] Bosutti A, Malaponte G, Zanetti M, et al. Calorie restriction modulates inactivity-induced changes in the inflammatory markers Creactive protein and pentraxin-3. Clin Endocrinol Metab 2008; 93(8): 3226-9.

[13] Araya AV, Orellana X, Espinoza J. Evaluation of the effect of caloric restriction on serum BDNF in overweight and obese subjects: preliminary evidences. Endocrine 2008; 33(3): 300-4.

[14] Heilbronn LK, de Jonge L, Frisard MI, et al. Pennington CALERIE Team. Effect of 6-month calorie restriction on biomarkers of longevity, metabolic adaptation, and oxidative stress in overweight individuals: a randomized controlled trial. JAMA 2006; 295(13): $1539-48$

[15] Redman LM, Ravussin E. Endocrine alterations in response to calorie restriction in humans. Mol Cell Endocrinol 2009; 299(1); 129-36.

[16] Weiss EP, Holloszy JO. Improvements in body composition, glucose tolerance, and insulin action induced by increasing energy expenditure or decreasing energy intake. J Nutr 2007; 137(4): 108790.

[17] Geng YQ, Guan JT, Xu MY, Xu XH, Fu YC. Behavioral study of calorie-restricted rats from early old age. Conf Proc IEEE Eng Med Biol Soc 2007; 2007: 2393-5.

[18] Witte AVM, Fobker R, Gellner S, Flöel KA. Caloric restriction improves memory in elderly humans. Proc Natl Acad Sci USA 2009 [Epub ahead of print].

[19] Karasik D, Demissie S, Cupples LA, Kiel DP. Disentangling the genetic determinants of human aging: biological age as an alternative to the use of survival measures. J Gerontol A Biol Sci Med Sci 2005; 60(5): 574-87.

[20] Ingram DK, Nakamura E, Smucny D, Roth GS, Lane MA. Strategy for identifying biomarkers of aging in long-lived species. Exp Gerontol 2001; 36(7): 1025-34.

[21] Anisimov VN, Berstein LM, Egormin PA, et al. Metformin slows down aging and extends life span of female SHR mice. Cell Cycle 2008; 7(17): 2769-73.

[22] Wood JG, Rogina B, Lavu S, et al. Sirtuin activators mimic caloric restriction and delay ageing in metazoans. Nature 2004; 430(7000): 686-9.

[23] Zyromski NJ, Mathur A, Pitt HA, et al. Cannabinoid receptor-1 blockade attenuates acute pancreatitis in obesity by an adiponectin mediated mechanism. J Gastrointest Surg 2009; 13(5): 831-8.

[24] Loh KY, Kew ST. Endocannabinoid system and cardio-metabolic risk. Med J Malaysia 2008; 63(4): 348-50.
[25] Sourris KC, Forbes JM, Cooper ME. Therapeutic interruption of advanced glycation in diabetic nephropathy: do all roads lead to Rome? Ann N Y Acad Sci 2008; 1126: 101-6.

[26] Tews D, Lehr S, Hartwig S, Osmers A, Paßlack W, Eckel J. Antiapoptotic action of exendin-4 in INS-1 beta cells: comparative protein pattern analysis of isolated mitochondria. Horm Metab Res 2009; 41(4): 294-301.

[27] Salgin B, Marcovecchio ML, Humphreys SM, et al. Effects of prolonged fasting and sustained lipolysis on insulin secretion and insulin sensitivity in normal subjects. Am J Physiol Endocrinol Metab 2009; 296(3): E454-61.

[28] Holguin F, Rojas M, Hart CM. The peroxisome proliferator activated receptor gamma (PPARgamma) ligand rosiglitazone modulates bronchoalveolar lavage levels of leptin, adiponectin, and inflammatory cytokines in lean and obese mice. Lung 2007; 185(6): 367-72.

[29] Nohr LA, Rasmussen LB, Straand J. Resin from the mukul myrrh tree, guggul, can it be used for treating hypercholesterolemia? A randomized, controlled study. Complement Ther Med 2009; 17(1): 16-22.

[30] Sirotkin AV, Chrenková M, Nitrayová S, et al. Effects of chronic food restriction and treatments with leptin or ghrelin on different reproductive parameters of male rats. Peptides 2008; 29(8): 1362-8.

[31] Majumdar P, Chen S, George B, Sen S, Karmazyn M, Chakrabarti $\mathrm{S}$. Leptin and endothelin-1 mediated increased extracellular matrix protein production and cardiomyocyte hypertrophy in diabetic heart disease. Diabetes Metab Res Rev 2009 [Epub ahead of print].

[32] Ingram DK, Zhu M, Mamczarz J, et al. Calorie restriction mimetics: an emerging research field. Aging Cell 2006; 5(2): 97-108.

[33] Gan L. Therapeutic potential of sirtuin-activating compounds in Alzheimer's disease. Drug News Perspect 2007; 20(4): 233-9.

[34] Kahyo T, Ichikawa S, Hatanaka T, Yamada MK, Setou M. A novel chalcone polyphenol inhibits the deacetylase activity of SIRT1 and cell growth in HEK293T cells. J Pharmacol Sci 2008; 108(3): 36471.

[35] Liu FC, Day YJ, Liou JT, Lau YT, Yu HP. Sirtinol attenuates hepatic injury and pro-inflammatory cytokine production following trauma-hemorrhage in male Sprague-Dawley rats. Acta Anaesthesiol Scand 2008; 52(5): 635-40.

[36] Lee JD, Huh JE, Jeon G, et al. Flavonol-rich RVHxR from Rhus verniciflua Stokes and its major compound fisetin inhibits inflammation-related cytokines and angiogenic factor in rheumatoid arthritic fibroblast-like synovial cells and in vivo models. Int Immunopharmacol 2009; 9(3): 268-76.

[37] Kang HL, Benzer S, Min KT. Life extension in Drosophila by feeding a drug. Proc Natl Acad Sci USA 2002; 99(2): 838-43.

[38] Vasques CA, Rossetto S, Halmenschlager G, et al. Evaluation of the pharmacotherapeutic efficacy of Garcinia cambogia plus Amorphophallus konjac for the treatment of obesity. Phytother Res 2008; 22(9): 1135-40.

[39] Hayamizu K, Tomi H, Kaneko I, Shen M, Soni MG, Yoshino G. Effects of Garcinia cambogia extract on serum sex hormones in overweight subjects. Fitoterapia 2008; 79(4): 255-61.

[40] Zhu XM, Xie P, Di YT, Peng SL, Ding LS, Wang MK. Two new triterpenoid saponins from Gymnema sylvestre. J Integr Plant Biol 2008; 50(5): 589-92.

[41] Kanaya AM, Harris T, Goodpaster BH, Tylavsky F, Cummings SR. Adipocytokines attenuate the association between visceral adiposity and diabetes in older adults; Health, Aging, and Body Composition (ABC) Study. Diabetes Care 2004; 27(6): 1375-80.

[42] Kondo M, Shibata R, Miura R, et al. Caloric restriction stimulates revascularization in response to ischemia via adiponectin-mediated activation of endothelial nitric-oxide synthase. J Biol Chem 2009; 284(3): 1718-24.

[43] Poehls J, Wassel CL, Harris TB, et al. Association of adiponectin with mortality in older adults: the Health, Aging, and Body Composition Study. Diabetologia 2009 [Epub ahead of print].

[44] Fawzia A Fahim, Mady EA. Antitumor activities of iodoacetate and dimethylsulphoxide against solid Ehrlich Carcinoma growth in mice. Biol Res 2003; 36 (2): 253-62.

[45] Siddiqui NI. Incretin mimetics and DPP-4 inhibitors: new approach to treatment of type 2 diabetes mellitus. Mymensingh Med J 2009; 18(1): 113-24.

[46] Karra E, Chandarana K, Batterham RL. The role of peptide YY in appetite regulation and obesity. J Physiol 2009; 587(Pt 1): 19-25. 
[47] Minor RK, Chang JW, de Cabo R. Hungry for life: How the arcuate nucleus and neuropeptide $\mathrm{Y}$ may play a critical role in mediating the benefits of calorie restriction. Mol Cell Endocrinol 2009; 299(1): 79-88.

[48] Wei M, Fabrizio P, Hu J, et al. Life span extension by calorie restriction depends on Rim15 and transcription factors downstream of Ras/PKA, Tor, and Sch9. PLoS Genet 2008; 4(1): e13.

[49] Tachibana T, Mori M, Khan MS, Ueda H, Sugahara K, Hiramatsu $\mathrm{K}$. Central administration of galanin stimulates feeding behavior in chicks. Comp Biochem Physiol A Mol Integr Physiol 2008 [Epub ahead of print].

[50] Vrontakis ME. Galanin: a biologically active peptide. Curr Drug Targets CNS Neurol Disord 2002; 1(6): 531-41.
[51] Caldeira da Silva CC, Cerqueira FM, Barbosa LF, Medeiros MH, Kowaltowski AJ. Mild mitochondrial uncoupling in mice affects energy metabolism, redox balance and longevity. Aging Cell 2008; 7(4): 552-60.

[52] Jonas WB, Ives JA. Should we explore the clinical utility of hormesis? Hum Exp Toxicol 2008; 27(2): 123-7.

[53] Kyriazis M. Clinical anti-aging hormetic strategies. Rejuvenation Res 2005; 8(2): 96-100.

[54] Honjoh S, Yamamoto T, Uno M, Nishida E. Signalling through RHEB-1 mediates intermittent fasting-induced longevity in C. elegans. Nature 2009; 457(7230): 726-30.

(C) Marios Kyriazis; Licensee Bentham Open.

This is an open access article licensed under the terms of the Creative Commons Attribution Non-Commercial License (http://creativecommons.org/licenses/by-nc/3.0/) which permits unrestricted, non-commercial use, distribution and reproduction in any medium, provided the work is properly cited. 